\title{
Conservation laws for even order elliptic systems in the critical dimension - a new approach
}

\author{
Jasmin Hörter ${ }^{1} \cdot$ Tobias Lamm $^{1}$ (D)
}

Received: 23 June 2020 / Accepted: 16 April 2021 / Published online: 25 June 2021

(c) The Author(s) 2021

\section{Abstract}

We consider elliptic systems of order $2 m$ in dimension $2 m$ which are generalizations of extrinsic and intrinsic polyharmonic maps. We show the existence of a conservation law for these systems by using a small perturbation of Uhlenbeck's gauge fixing matrix.

\section{Introduction}

The regularity of critical points of geometric variational problems for maps between two Riemannian manifolds attracted a lot of attention over the last two decades. The most prominent example are the harmonic maps which are critical points $u \in W^{1,2}(M, N)$ of the Dirichlet energy

$$
E(u)=\frac{1}{2} \int_{M}|\nabla u|^{2} d v_{g},
$$

where $(M, g)$ and $(N, h)$ are two smooth and compact manifolds without boundary and $N$ is isometrically embedded into some euclidean space $\mathbb{R}^{n}$. They solve the elliptic system

$$
-\Delta u=A(u)(\nabla u, \nabla u)
$$

where $A$ is the second fundamental form of the embedding $N \hookrightarrow \mathbb{R}^{n}$. The Dirichlet energy is scaling invariant in dimension two, which is called the critical dimension, and it was shown by Hélein [10] that weakly harmonic maps are smooth in this case.

Communicated by A. Mondino.

The authors acknowledge funding by the Deutsche Forschungsgemeinschaft (DFG, German Research Foundation) - 281869850 (RTG 2229).

Tobias Lamm

tobias.lamm@kit.edu

Jasmin Hörter

jasmin.hoerter@kit.edu

1 Department of Mathematics, Karlsruhe Institute of Technology, 76128 Karlsruhe, Germany 
This result was substantially extended by Rivière [15] to more general elliptic systems of the form

$$
-\Delta u=\Omega \cdot \nabla u
$$

where $\Omega \in L^{2}\left(B^{2}, s o(n) \otimes \wedge^{1}\left(\mathbb{R}^{2}\right)\right)$ and $B^{2}$ denotes the unit ball in $\mathbb{R}^{2}$. Rivière obtained the regularity of weak solutions as a consequence of a conservation law which he derived using the antisymmetry of $\Omega$. The key ingredient here was the use of the Uhlenbeck gauge fixing result [20], see Theorem 3.2. Note that the Euler-Lagrange equation of all quadratic and conformally invariant variational integrals satisfies an equation of the type above. We sketch a version of this result in Sect. 3 .

The regularity result of Hélein was than extended to the so-called weakly biharmonic maps in $\mathbb{R}^{4}$, i.e. critical points of the functional

$$
E_{2}(u)=\frac{1}{2} \int_{M}|\Delta u|^{2} d v_{g}
$$

by Chang-Wang-Yang [3] for spherical targets and by Wang [21] for general targets. Later, the second author and Rivière [14] were able to show a conservation law for a suitable generalization of the biharmonic map equation in the spirit of the before mentioned paper of Rivière. A modified version of this conservation law was later obtained by Struwe [18].

De Longueville and Gastel [5] recently extended this result to systems of order $2 m$ in the critical dimension. The motivating example behind this system are the $m$-polyharmonic maps $u \in W^{m, 2}\left(B^{2 m}, N\right)$, which are critical points of the functional

$$
E_{m}(u)=\frac{1}{2} \int_{B^{2 m}}\left|\nabla^{m} u\right|^{2} d v_{g} .
$$

The Euler-Lagrange equation for $E_{m}$ was calculated by Angelsberg-Pumberger [1] resp. Gastel-Scheven [6]. In the latter paper the authors also showed the regularity for these critical points using Hélein's moving frame technique.

In the following we consider systems of the from

$$
\Delta^{m} u=\sum_{k=0}^{m-1} \Delta^{k}\left\langle V_{k}, d u\right\rangle+\sum_{k=0}^{m-2} \Delta^{k} \delta\left(w_{k} d u\right) .
$$

with coefficient functions

$$
\begin{array}{ll}
w_{k} \in W^{2 k+2-m, 2}\left(B^{2 m}, \mathbb{R}^{n \times n}\right) & \text { for } k \in\{0, \ldots, m-2\}, \\
V_{k} \in W^{2 k+1-m, 2}\left(B^{2 m}, \mathbb{R}^{n \times n} \otimes \wedge^{1} \mathbb{R}^{2 m}\right) & \text { for } k \in\{0, \ldots, m-1\}, \text { where } \\
V_{0}=d \eta+F, \quad \eta \in W^{2-m, 2}\left(B^{2 m}, \operatorname{so}(n)\right), & F \in W^{2-m, \frac{2 m}{m+1}, 1}\left(B^{2 m}, \mathbb{R}^{n \times n} \otimes \wedge^{1} \mathbb{R}^{2 m}\right) .
\end{array}
$$

It was shown by De Longueville and Gastel [5] that $m$-polyharminic maps are solutions of a system of this type. Note that the definition and the basic properties of the negative Sobolev spaces arising in this equation are collected in Sect. 2.

In our main Theorem 2.13 we establish a new conservation law for systems of the form (1.1). The novelty here is that we use a small perturbation of the gauge fixing matrix $P$ in a suitable variant of the Uhlenbeck result, see Theorem 4.1.

The paper is organized as follows. In Sect. 2 we recall some basic definitions and properties for negative Sobolev and Lorentz-Sobolev spaces and we show a suitable higher order generalization of the Wente Lemma. Moreover, we state and comment on our main Theorem. 
In Sect. 3 we review the second order case of our main result, a proof of which was already sketched by Rivière in [16].

In Sect. 4 we finally show our main Theorem.

\section{Lorentz-Sobolev spaces and the main result}

In this section we start by recalling the definitions of the relevant function spaces we need in order to obtain the desired conservation law. Moreover, we show a preliminary result on a higher order version of the famous Wente lemma [22] and we state our main result.

\subsection{Lorentz- and Lorentz-Sobolev spaces}

Important function spaces in our paper are the so called Lorentz spaces. They are interpolation spaces of the classical $L^{p}$-spaces and in the following we briefly collect a few properties of these spaces. For detailed proofs see for example $[5,8,11,12,19,23]$. We start with a Lemma on the Hölder inequality for these functions

Lemma 2.1 (Hölder inequality) Let $f \in L^{p_{1}, q_{1}}\left(\mathbb{R}^{n}\right)$ and $g \in L^{p_{2}, q_{2}}\left(\mathbb{R}^{n}\right)$ with $\frac{1}{p_{1}}+\frac{1}{p_{2}}=$ $\frac{1}{p}, \frac{1}{q_{1}}+\frac{1}{q_{2}}=\frac{1}{q}$ and $p_{1}, p_{2} \in(1, \infty), q_{1}, q_{2} \in[1, \infty]$. Then

$$
\|f g\|_{L^{p, q}\left(\mathbb{R}^{n}\right)} \leq\|f\|_{L^{p_{1}, q_{1}}\left(\mathbb{R}^{n}\right)}\|g\|_{L^{p_{2}, q_{2}}\left(\mathbb{R}^{n}\right)} .
$$

Additionally, we also need the following estimates.

Lemma 2.2 Let $f: \mathbb{R}^{n} \rightarrow \mathbb{R}$ be measurable.

1. Let $1<p \leq \infty$ and $1 \leq q<Q \leq \infty$. Then we have

$$
\|f\|_{L^{p, Q}\left(\mathbb{R}^{n}\right)} \leq c\|f\|_{L^{p, q}\left(\mathbb{R}^{n}\right)} .
$$

2. Let $1<p<P \leq \infty, 1 \leq q_{1}, q_{2} \leq \infty$ and let $\Omega \subset \mathbb{R}^{n}$ be bounded. Then we have

$$
\|f\|_{L^{p, q_{1}}(\Omega)} \leq c|\Omega|^{\frac{1}{p}-\frac{1}{P}}\|f\|_{L^{P, q_{2}(\Omega)}} .
$$

Next we come to Lorentz-Sobolev spaces. If a function $f \in L^{p, q}\left(\mathbb{R}^{n}\right)$ has derivatives $D^{j} f \in L^{p, q}\left(\mathbb{R}^{n}\right)$ for all $1 \leq j \leq k \in \mathbb{N}$, then $f$ is an element of the so-called LorentzSobolev space $W^{k, p, q}\left(\mathbb{R}^{n}\right)$.

Definition 2.3 Let $1<p \leq \infty, 1 \leq q \leq \infty$ and $k \in \mathbb{N}$. Let $f \in L^{p, q}\left(\mathbb{R}^{n}\right)$ be $k$ times weakly differentiable and for all multiindices $\alpha \in \mathbb{N}_{0}^{n}$ with $|\alpha| \leq k$ let $\frac{\partial^{|\alpha|}}{\partial^{\alpha} 1 x_{1} \ldots \partial^{\alpha_{n}} x_{n}} f \in L^{p, q}\left(\mathbb{R}^{n}\right)$. Then $f$ is an element of the Lorentz-Sobolev space $W^{k, p, q}\left(\mathbb{R}^{n}\right)$ with norm

$$
\|f\|_{W^{k, p, q}\left(\mathbb{R}^{n}\right)}:=\sum_{0 \leq|\alpha| \leq k}\left\|\frac{\partial^{|\alpha|}}{\partial^{\alpha_{1}} x_{1} \ldots \partial^{\alpha_{n}} x_{n}} f\right\|_{L^{p, q\left(\mathbb{R}^{n}\right)}} .
$$

We have a generalized Sobolev embedding theorem for these spaces.

Lemma 2.4 Let $k, n \in \mathbb{N}, 1<p<\frac{n}{k}$ and $1 \leq q \leq \infty$. Then

$$
W^{k, p, q}\left(B^{n}\right) \hookrightarrow L^{p^{*}, q}\left(B^{n}\right)
$$

for $\frac{1}{p^{*}}=\frac{1}{p}+\frac{k}{n}$ with the estimate

$$
\|f\|_{L^{p^{*}, q}\left(B^{n}\right)} \leq c \|\left. f\right|_{W^{k, p, q}\left(B^{n}\right)} \quad \text { for any } f \in W^{k, p, q}\left(B^{n}\right) .
$$


Similar to Lemma 2.1 we have a product estimate for Lorentz-Sobolev functions.

Lemma 2.5 Let $s, k \in \mathbb{N}, p, p^{\prime}, q, q^{\prime} \in \mathbb{R}$ with $1<p, p^{\prime}, q, q^{\prime}<\infty, k p<n, s p^{\prime}<$ $n, s \leq k, t:=\frac{n p p^{\prime}}{n\left(p+p^{\prime}\right)-k p p^{\prime}}>1$ and $\frac{1}{u}:=\min \left\{\frac{1}{q}+\frac{1}{q^{\prime}}, 1\right\}$. Further let $B^{n} \subset \mathbb{R}^{n}$. If $f \in W^{k, p, q}\left(B^{n}\right), g \in W^{s, p^{\prime}, q^{\prime}}\left(B^{n}\right)$, then

$$
f g \in W^{s, t, u}\left(B^{n}\right)
$$

and

$$
\|f g\|_{W^{s, t, u}\left(B^{n}\right)} \leq c\|f\|_{W^{k, p, q}\left(B^{n}\right)}\|g\|_{W^{s, p^{\prime} q^{\prime}}\left(B^{n}\right)}
$$

with $c=c\left(B^{n}\right)$.

Furthermore, we need an optimal Sobolev embedding result.

Lemma 2.6 Let $B^{n} \subset \mathbb{R}^{n}$. If $f \in W^{k, \frac{n}{k}, 1}\left(B^{n}\right)$, then $f$ is continuous on $B^{n}$.

Later on we also use Lorentz-Sobolev spaces $W^{-k, p^{\prime}, q^{\prime}}$. These are distribution spaces and for $p, q>1$ they are the dual spaces of $W^{k, p, q}$.

Definition 2.7 Let $1<p, q<\infty, \frac{1}{p}+\frac{1}{p^{\prime}}=\frac{1}{q}+\frac{1}{q^{\prime}}=1$ and $k \in \mathbb{N}$. Then $W^{-k, p, q}\left(B^{n}\right)$ is the space of distributions $\Phi \in\left(C_{c}^{\infty}\left(B^{n}\right)\right)^{\prime}$ such that

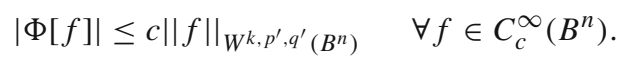

Each element of $W^{-k, p, q}$ has a representation in terms of derivatives of Lorentz functions:

Lemma 2.8 Let $1<p, q<\infty, k \in \mathbb{N}, B^{n} \subset \mathbb{R}^{n}$ and $f \in W^{-k, p, q}\left(B^{n}\right)$. Then there exist $f_{\alpha} \in L^{p, q}\left(B^{n}\right)$ so that

$$
f=\sum_{|\alpha| \leq k} \partial^{\alpha} f_{\alpha}
$$

Note that this representation is not unique. We define the norm on $W^{-k, p, q}\left(B^{n}\right)$ by

$$
\|f\|_{W^{-k, p, q\left(B^{n}\right)}}:=\inf \left\{\sum_{|\alpha| \leq k}\left\|f_{\alpha}\right\|_{L^{p, q}\left(B^{n}\right)}: f=\sum_{|\alpha| \leq k} \partial^{\alpha} f_{\alpha}\right\} .
$$

The definition of negative Lorentz-Sobolev spaces as dual spaces does not hold for $p, q=$ 1 since $L^{p, 1}, L^{p^{\prime}, \infty}$ are not reflexive. In this case we define the space $W^{-k, p, 1}$ as follows

Definition 2.9 Let $1<p<\infty, k \in \mathbb{N}$. Then

$$
W^{-k, p, 1}\left(B^{n}\right):=\left\{f=\sum_{|\alpha| \leq k} \partial^{\alpha} f_{\alpha}: f_{\alpha} \in L^{p, 1}\left(B^{n}\right)\right\}
$$

with norm

$$
\|f\|_{W^{-k, p, 1}\left(B^{n}\right)}:=\inf \left\{\sum_{|\alpha| \leq k}\left\|f_{\alpha}\right\|_{L^{p, 1}\left(B^{n}\right)}: f=\sum_{|\alpha| \leq k} \partial^{\alpha} f_{\alpha}\right\}
$$

Finally we have an embedding theorem and a Hölder inequality. 
Lemma 2.10 Let $B^{n} \subset \mathbb{R}^{n}, 1<p<n, 1 \leq q \leq p, l, s, t \in \mathbb{N}_{0}$ with tp $<n$ and $f \in W^{-s, p, q}\left(B^{n}, \wedge^{l} \mathbb{R}^{n}\right)$. Then $f \in W^{-(s+t), \frac{n p}{n-t p}, q}\left(B^{n}, \wedge^{l} \mathbb{R}^{n}\right)$ and

$$
\|f\|_{W^{-(s+t), \frac{n p}{n-t p}, q}\left(B^{n}\right)} \leq c\|f\|_{W^{-s, p, q}\left(B^{n}\right)} .
$$

Lemma 2.11 Let $s, t \in \mathbb{N}, t \leq s, 1<p, p^{\prime}<\infty$ with $\frac{1}{p}+\frac{1}{p^{\prime}} \leq 1$ and $t p<n$, $s p^{\prime}<$ $n, 1 \leq q, q^{\prime}<\infty$. Let $f \in W^{-t, p, q}\left(B^{n}\right)$ and $g \in W^{s, p^{\prime}, q^{\prime}}\left(B^{n}\right)$. Then

$$
f g \in W^{-t, x, y}\left(B^{n}\right)
$$

with $x=\frac{d p p^{\prime}}{n\left(p+p^{\prime}\right)-s p p^{\prime}}$ and $\frac{1}{y}=\min \left\{1, \frac{1}{q}, \frac{1}{q^{\prime}}\right\}$. Further

$$
\|f g\|_{W^{-t, x, y}\left(B^{n}\right)} \leq c\|f\|_{W^{-t, p, q}\left(B^{n}\right)}\|g\|_{W^{s, p^{\prime}, q^{\prime}\left(B^{n}\right)}} .
$$

More details about these spaces and proofs of the above results can be found in [4].

\subsection{A generalized Wente lemma}

A key ingredient in the proof of the main Theorem later on will be the following Wente-type lemma in the spirit of Bethuel and Ghidaglia [2]. A fourth order version of this result can already be found in [14].

Lemma 2.12 Let $\sigma>0, f \in L^{\frac{2 m}{2 m-1-|\gamma|}, 1}\left(B^{2 m}, \mathbb{R}^{n}\right)$ for $|\gamma| \leq m-2$ and $P \in$ $W^{m, 2}\left(B^{2 m}, S O(n)\right)$ with $\|d P\|_{W^{m-1,2}} \leq \sigma$. There exists $\sigma_{0}>0$ such that if $\sigma<\sigma_{0}$ there exists a unique solution $u \in W^{2 m-1, \frac{2 m}{2 m-1-|\gamma|}, 1}\left(B^{2 m}, M(n)\right)$ of

$$
\left\{\begin{array}{ccc}
\Delta\left(\Delta^{m-1} u \cdot P\right) & =\delta f & \text { in } B^{2 m}, \\
\Delta^{j} u & =0 & \text { on } \partial B^{2 m} \text { for } j=0, \ldots, m-1,
\end{array}\right.
$$

with

$$
\left\|D^{2 m-1} u\right\|_{L^{\frac{2 m}{2 m-1-|\gamma|}, 1}\left(B^{2 m}\right)}+\|u\|_{L^{\infty}\left(B^{2 m}\right)} \leq c\|f\|_{L^{\frac{2 m}{2 m-1-|\gamma|}, 1}\left(B^{2 m}\right)} \cdot
$$

Proof The boundary conditions determine a solution $u$ of (2.1) uniquely. To see this we assume there exist solutions $u_{1}, u_{2}$ and we let $v:=u_{1}-u_{2}$. Then $\Delta\left(\Delta^{m-1} v \cdot P\right)=0$. Testing this equation with $\Delta^{m-1} v \cdot P$ and integrating by parts gives

$$
0=\int_{B^{2 m}} \Delta\left(\Delta^{m-1} v \cdot P\right)\left(\Delta^{m-1} v \cdot P\right)=-\int_{B^{2 m}}\left|D\left(\Delta^{m-1} v \cdot P\right)\right|^{2} .
$$

Thus we have $D\left(\Delta^{m-1} v \cdot P\right)=0$ and therefore $\Delta^{m-1} v \cdot P=$ const. Because $P$ is invertible and $\Delta^{m-1} v=0$ on $\partial B^{2 m}$ we get $\Delta^{m-1} v=0$. Iteratively we get $v=0$ and thus $u_{1}=u_{2}$.

Now we approximate $f$ by $\bar{f} \in C_{c}^{\infty}\left(\mathbb{R}^{2 m}\right)$ so that $\bar{f}=0$ on $\mathbb{R}^{2 m} \backslash B^{2 m}$ and

$$
\|\bar{f}\|_{L^{\frac{2 m}{2 m-1-|\gamma|}, 1}\left(\mathbb{R}^{2 m}\right)} \leq c\|f\|_{L^{\frac{2 m}{2 m-1-|\gamma|}},{ }_{\left(B^{2 m}\right)}} .
$$

Standard $L^{p}$-theory and interpolation results (see [11] Theorem 3.3.3) yield

$$
\left\|D\left(\Delta^{m-1} u P\right)\right\|_{L^{\frac{2 m}{2 m-1-|\gamma|}, 1}\left(B^{2 m}\right)} \leq c\|f\|_{L^{\frac{2 m}{2 m-1-|\gamma|}, 1}\left(B^{2 m}\right)} .
$$


With Hölder's inequality for Lorentz spaces and the embedding theorem we estimate

$$
\begin{aligned}
& \left\|D \Delta^{m-1} u\right\| L_{L^{\frac{2 m}{2 m-1-|\gamma|}, 1}\left(B^{2 m}\right)} \\
& \quad \leq c\left(\|f\|_{L^{\frac{2 m}{2 m-1-|\gamma|}, 1}\left(B^{2 m}\right)}+\left\|D^{2 m-2} u\right\|_{L^{\frac{2 m}{2 m-2-|\gamma|}, 2}\left(B^{2 m}\right)}\|d P\|_{L^{2 m, 2}\left(B^{2 m}\right)}\right) \\
& \quad \leq c\left(\|f\|_{L^{\frac{2 m}{2 m-1-|\gamma|}, 1}\left(B^{2 m}\right)}+\|u\|_{W^{2 m-1, \frac{2 m}{2 m-1-|\gamma|}, 1}\left(B^{2 m}\right)}\|d P\|_{W^{m-1,2}\left(B^{2 m}\right)}\right) .
\end{aligned}
$$

We interchange derivatives and apply the Calderon-Zygmund inequality

$$
\begin{aligned}
& \left\|D^{2 m-1} u\right\| \|_{L^{\frac{2 m}{2 m-1-|\gamma|}, 1}\left(B^{2 m}\right)} \\
& \quad \leq c\left(\|f\|_{L^{\frac{2 m}{2 m-1-|\gamma|}, 1}\left(B^{2 m}\right)}+\|u\|_{W^{2 m-1, \frac{2 m}{2 m-1-|\gamma|}, 1}\left(B^{2 m}\right)}\|d P\|_{W^{m-1,2}\left(B^{2 m}\right)}\right) .
\end{aligned}
$$

Since $\|d P\|_{W^{m-1,2}\left(B^{2 m}\right)}<\sigma$ we absorb the second term to the left-hand side. The density of $C_{c}^{\infty}\left(B^{2 m}\right)$ in $L^{p, q}\left(B^{2 m}\right)$ finishes the proof.

\subsection{The main result}

Before we are able to state our main result we introduce some more notation. Let $\wedge^{k} \mathbb{R}^{2 m}, k \in$ $\mathbb{N}_{0}$ be the space of $k$-forms on $\mathbb{R}^{2 m}$. Further let

$$
d: W^{1, p}\left(\mathbb{R}^{2 m}, \wedge^{k} \mathbb{R}^{2 m}\right) \rightarrow L^{p}\left(\mathbb{R}^{2 m}, \wedge^{k+1} \mathbb{R}^{2 m}\right)
$$

be the exterior derivative and

$$
\delta: W^{1, p}\left(\mathbb{R}^{2 m}, \wedge^{k} \mathbb{R}^{2 m}\right) \rightarrow L^{p}\left(\mathbb{R}^{2 m}, \wedge^{k-1} \mathbb{R}^{2 m}\right)
$$

the codifferential. We have $d d=\delta \delta=0$ and the Laplacian is given by

$$
\Delta=d \delta+\delta d
$$

If $f$ is a function, the exterior derivative of $f$ is just the gradient $\nabla f$. Let $0 \leq k \leq 2 m$ with $k \in \mathbb{N}$, then we let

$$
*: \wedge^{k} \mathbb{R}^{2 m} \rightarrow \wedge^{2 m-k} \mathbb{R}^{2 m}
$$

be the Hodge-Star operator. For a $k$-form $\omega$ we have

$$
\delta \omega=(-1)^{2 m(k+1)+1} * d * \omega
$$

and

$$
\text { ** }:(-1)^{k(2 m-k)}: \wedge^{k} \mathbb{R}^{2 m} \rightarrow \wedge^{k} \mathbb{R}^{2 m} .
$$

(see e.g. [13]).

The following is the main result of this paper.

Theorem 2.13 Assume $m \geq 2, n \in \mathbb{N}$. Let coefficient functions be given as

$$
\begin{aligned}
& w_{k} \in W^{2 k+2-m, 2}\left(B^{2 m}, \mathbb{R}^{n \times n}\right) \quad \text { for } k \in\{0, \ldots, m-2\}, \\
& V_{k} \in W^{2 k+1-m, 2}\left(B^{2 m}, \mathbb{R}^{n \times n} \otimes \wedge^{1} \mathbb{R}^{2 m}\right) \quad \text { for } k \in\{0, \ldots, m-1\}, \text { where } \\
& V_{0}=d \eta+F, \quad \eta \in W^{2-m, 2}\left(B^{2 m}, \operatorname{so}(n)\right), \quad F \in W^{2-m, \frac{2 m}{m+1}, 1}\left(B^{2 m}, \mathbb{R}^{n \times n} \otimes \wedge^{1} \mathbb{R}^{2 m}\right)
\end{aligned}
$$


We consider the equation

$$
\Delta^{m} u=\sum_{k=0}^{m-1} \Delta^{k}\left\langle V_{k}, d u\right\rangle+\sum_{k=0}^{m-2} \Delta^{k} \delta\left(w_{k} d u\right)
$$

For this equation, the following statements hold.

(i) Let

$$
\begin{aligned}
\sigma:= & \sum_{k=0}^{m-2}\left\|w_{k}\right\|_{W^{2 k+2-m, 2}\left(B^{2 m}\right)}+\sum_{k=1}^{m-1}\left\|V_{k}\right\|_{W^{2 k+1-m, 2}\left(B^{2 m}\right)} \\
& +\|\eta\|_{W^{2-m, 2}\left(B^{2 m}\right)}+\|F\|_{W^{2-m, \frac{2 m}{m+1}, 1}\left(B^{2 m}\right)} .
\end{aligned}
$$

There is $\sigma_{0}>0$ such that whenever $\sigma<\sigma_{0}$, there exist $\varepsilon \in W^{m, 2} \cap L^{\infty}\left(B_{1 / 2}^{2 m} ; M(n)\right)$ with

$$
\|\varepsilon\|_{W^{m, 2}\left(B_{1 / 2}^{2 m}\right)}+\|\varepsilon\|_{L^{\infty}\left(B_{1 / 2}^{2 m}\right)} \leq c \sigma,
$$

a function $P \in W^{m, 2}\left(B_{1 / 2} ; S O(n)\right)$ and a distribution $B \in W_{l o c}^{2-m, 2}\left(B_{1 / 2}^{2 m}, \mathbb{R}^{n \times n} \otimes\right.$ $\wedge^{2} \mathbb{R}^{2 m}$ ) which solves

$$
\delta B=\sum_{k=0}^{m-1} \Delta^{k}((i d+\varepsilon) P) V_{k}-\sum_{k=0}^{m-2} d \Delta^{k}((i d+\varepsilon) P) w_{k}+d \Delta^{m-1}((i d+\varepsilon) P) .
$$

(ii) A function $u \in W^{m, 2}\left(B_{1 / 2}^{2 m}, \mathbb{R}^{n}\right)$ solves (2.4) weakly if and only if it is a distributional solution of the conservation law

$$
\begin{aligned}
& \delta\left[\sum_{l=0}^{m-1} \Delta^{l}((i d+\varepsilon) P) \Delta^{m-l-1} d u-\sum_{l=0}^{m-2} d \Delta^{l}((i d+\varepsilon) P) \Delta^{m-l-1} u\right. \\
& \quad-\sum_{k=0}^{m-1} \sum_{l=0}^{k-1} \Delta^{l}((i d+\varepsilon) P) \Delta^{k-l-1} d\left\langle V_{k}, d u\right\rangle \\
& \quad+\sum_{k=0}^{m-1} \sum_{l=0}^{k-1} d \Delta^{l}((i d+\varepsilon) P) \Delta^{k-l-1}\left\langle V_{k}, d u\right\rangle \\
& \quad-\sum_{k=0}^{m-2} \sum_{l=0}^{k} \Delta^{l}((i d+\varepsilon) P) d \Delta^{k-l-1} \delta\left(w_{k} d u\right) \\
& \left.\quad+\sum_{k=0}^{m-2} \sum_{l=0}^{k-1} d \Delta^{l}((i d+\varepsilon) P) \Delta^{k-l-1} \delta\left(w_{k} d u\right)-\langle B, d u\rangle\right]=0 .
\end{aligned}
$$

(iii) Every weak solution $u$ of (2.4) is continuous.

A different variant of this result has been obtained earlier by Lamm and Rivière [14] in the case $m=2$ and by De Longueville and Gastel [5] for general $m$. The key difference to these papers is that we use a small perturbation $(i d+\varepsilon) P$ of the Uhlenbeck gauge matrix $P$, see Theorem 4.1, to establish the conservation law. This Ansatz highlights the strong connection between the conservation law and the matrix $P$ more explicitly than the previous papers. Another new ingredient in our approach is Lemma 2.12, a generalization of an estimate by 
Bethuel and Ghidaglia [2], which we use instead of a Wente type result for the poly-Laplace operator. This allows for more general elliptic operators in divergence form and simplifies the argument.

We also remark that in a recent paper by Guo and Xiang [9] it was shown that weak solutions of (2.4) are not only continuous but even Hölder continuous for some positive exponent.

\section{Second order case}

In this section we briefly review the second order case of the main Theorem 2.13 . We will not discuss the original proof in [15] but we will focus on Rivière's subsequent idea to establish a conservation law by using a small perturbation of the Uhlenbeck gauge matrix $P$. This proof was already sketched in [16], chapter VI, but since we will follow the same strategy in the proof of our main Theorem we decided to include this argument here.

Theorem 3.1 Let $n \in \mathbb{N}$ and $N$ be an oriented submanifold of $\mathbb{R}^{n}$. Let $u \in W^{1,2}\left(B^{2}, N\right)$ be a solution of

$$
-\Delta u=\Omega \cdot \nabla u,
$$

where $\Omega \in L^{2}\left(B^{2}, \operatorname{so}(n) \otimes \wedge^{1} \mathbb{R}^{2}\right)$ and let $\sigma:=\|\Omega\|_{L^{2}}$. There exists $\sigma_{0}>0$ such that whenever $\sigma<\sigma_{0}$, there exist $\varepsilon \in W^{1,2} \cap L^{\infty}\left(B^{2}, M(n)\right), P \in W^{1,2}\left(B^{2}, S O(n)\right)$ and $\xi \in W^{1,2}\left(B^{2}, \operatorname{so}(n)\right)$ with

$$
\|\varepsilon\|_{L^{\infty}\left(B^{2}\right)}+\|\nabla \varepsilon\|_{L^{2}\left(B^{2}\right)}+\|\xi\|_{W^{1,2}\left(B^{2}\right)}+\|\nabla P\|_{L^{2}\left(B^{2}\right)} \leq c \sigma,
$$

and $B \in W^{1,2}\left(B^{2}\right)$ that solve

$$
\nabla^{\perp} B=\nabla \varepsilon P-(i d+\varepsilon) \nabla^{\perp} \xi P .
$$

Further $u$ solves (3.1) if and only if it is a solution of

$$
-\operatorname{div}((i d+\varepsilon) P \nabla u)=\nabla^{\perp} B \cdot \nabla u
$$

and $u$ is continuous.

The proof of Theorem 3.1 relies heavily on Uhlenbeck's gauge theorem, see for example $[15,17,20]$.

Theorem 3.2 [Uhlenbeck gauge] There exists $\sigma>0$ and $c>0$ such that for every $\Omega \in$ $L^{2}\left(B^{2}\right.$, so $\left.(n) \otimes \wedge^{1} \mathbb{R}^{2}\right)$ satisfying $\|\Omega\|_{L^{2}\left(B^{2}\right)}<\sigma$ there exist $P \in W^{1,2}\left(B^{2}, S O(n)\right)$ and $\xi \in W^{1,2}\left(B^{2}\right.$, so $\left.(n)\right)$ such that

$$
\Omega=P^{-1} \nabla^{\perp} \xi P+P^{-1} \nabla P
$$

and

$$
\|\xi\|_{W^{1,2}\left(B^{2}\right)}+\|\nabla P\|_{L^{2}\left(B^{2}\right)} \leq c\|\Omega\|_{L^{2}\left(B^{2}\right)} .
$$

Proof of Theorem 3.1: Assume $\|\Omega\|_{L^{2}\left(B^{2}\right)}<\sigma$ as in Theorem 3.2. Then we get $P \in$ $W^{1,2}\left(B^{2}, S O(n)\right), \xi \in W^{1,2}\left(B^{2}, \operatorname{so}(n)\right)$ such that

$$
\begin{aligned}
& \Omega=P^{-1} \nabla^{\perp} \xi P+P^{-1} \nabla P \text { and } \\
& \quad\|\xi\|_{W^{1,2}\left(B^{2}\right)}+\|\nabla P\|_{L^{2}\left(B^{2}\right)} \leq c\|\Omega\|_{L^{2}\left(B^{2}\right)} .
\end{aligned}
$$


We multiply (3.1) with $(i d+\varepsilon) P$, where $\varepsilon \in W^{1,2} \cap L^{\infty}\left(B^{2}, M(n)\right)$ and $i d$ is the identity matrix in $\mathbb{R}^{n}$, and obtain

$$
\begin{aligned}
-(i d+\varepsilon) P \Delta u & =(i d+\varepsilon) P \Omega \cdot \nabla u \\
\Leftrightarrow-\operatorname{div}[(i d+\varepsilon) P \nabla u] & =[-\nabla \varepsilon P+(i d+\varepsilon)(-\nabla P+P \Omega)] \cdot \nabla u \\
\Leftrightarrow-\operatorname{div}[(i d+\varepsilon) P \nabla u] & =\left[-\nabla \varepsilon P+(i d+\varepsilon) \nabla^{\perp} \xi P\right] \cdot \nabla u .
\end{aligned}
$$

We choose $\varepsilon \in W^{1,2} \cap L^{\infty}\left(B^{2}, M(n)\right)$ such that

$$
\operatorname{div}\left[-\nabla \varepsilon P+(i d+\varepsilon) \nabla^{\perp} \xi P\right]=0 .
$$

To do this we apply a fixed point argument. Let

$$
\begin{aligned}
\psi: W^{1,2} \cap L^{\infty}\left(B^{2}\right) & \rightarrow W^{1,2} \cap L^{\infty}\left(B^{2}\right) \\
\varepsilon & \mapsto \text { solution } \lambda \text { of }(3.4)
\end{aligned}
$$

where

$$
\left\{\begin{array}{rlrl}
\operatorname{div}[\nabla \lambda P] & =\nabla((i d+\varepsilon) P) \cdot \nabla^{\perp} \xi & & \text { in } B^{2}, \\
\lambda=0 & \text { on } \partial B^{2} .
\end{array}\right.
$$

Let $\varepsilon_{1}, \varepsilon_{2} \in W^{1,2} \cap L^{\infty}\left(B^{2}\right)$ and $\psi\left(\varepsilon_{1}\right)=\lambda_{1}, \psi\left(\varepsilon_{2}\right)=\lambda_{2}$ be the corresponding solutions of (3.4). Then $\Lambda:=\lambda_{1}-\lambda_{2}$ solves

$$
\left\{\begin{aligned}
\operatorname{div}[\nabla \Lambda P] & =\nabla\left(\left(\varepsilon_{1}-\varepsilon_{2}\right) P\right) \cdot \nabla^{\perp} \xi & & \text { in } B^{2}, \\
\Lambda & =0 & & \text { on } \partial B^{2} .
\end{aligned}\right.
$$

Since $P$ takes values in $S O(n)$ it satisfies the assumptions of Theorem 1.3 in [2] and we have

$$
\begin{aligned}
\|\Lambda\|_{L^{\infty}\left(B^{2}\right)}+\|\nabla \Lambda\|_{L^{2}\left(B^{2}\right)} \leq & c\left(\left\|\nabla \varepsilon_{1}-\nabla \varepsilon_{2}\right\|_{L^{2}\left(B^{2}\right)}\|P\|_{L^{\infty}\left(B^{2}\right)}\right. \\
& \left.+\left\|\varepsilon_{1}-\varepsilon_{2}\right\|_{L^{\infty}\left(B^{2}\right)}\|\nabla P\|_{L^{2}\left(B^{2}\right)}\right) \cdot\|\nabla \xi\|_{L^{2}\left(B^{2}\right)} \\
\leq & c \sigma\left(\left\|\nabla \varepsilon_{1}-\nabla \varepsilon_{2}\right\|_{L^{2}\left(B^{2}\right)}+\left\|\varepsilon_{1}-\varepsilon_{2}\right\|_{L^{\infty}\left(B^{2}\right)}\right) .
\end{aligned}
$$

For $\sigma$ small enough we conclude that $\psi$ is a contraction. To show that $\psi$ is a self-map from a small ball in $W^{1,2} \cap L^{\infty}\left(B^{2}\right)$ into itself, we use again Theorem 1.3 in [2] to get

$$
\begin{aligned}
\|\lambda\|_{L^{\infty}\left(B^{2}\right)}+\|\nabla \lambda\|_{L^{2}\left(B^{2}\right)} \leq & c\|\nabla \xi\|_{L^{2}\left(B^{2}\right)}\left(\|\nabla \varepsilon\|_{L^{2}\left(B^{2}\right)}\right. \\
& \left.+\left(1+\|\varepsilon\|_{L^{\infty}\left(B^{2}\right)}\right)\|\nabla P\|_{L^{2}\left(B^{2}\right)}\right) .
\end{aligned}
$$

The Banach fixed point theorem yields a unique $\varepsilon^{*} \in W^{1,2} \cap L^{\infty}\left(B^{2}, M(n)\right)$ solving (3.4) and hence also (3.3) and with the estiamte above we get

$$
\left\|\varepsilon^{*}\right\|_{L^{\infty}\left(B^{2}\right)}+\left\|\nabla \varepsilon^{*}\right\|_{L^{2}\left(B^{2}\right)} \leq c \sigma .
$$

By the Poincaré lemma there exists $B \in W^{1,2}\left(B^{2}\right)$ such that

$$
\nabla^{\perp} B=-\nabla \varepsilon^{*} P+\left(i d+\varepsilon^{*}\right) \nabla^{\perp} \xi P
$$

and (3.1) is equivalent to

$$
-\operatorname{div}\left(\left(i d+\varepsilon^{*}\right) P \nabla u\right)=\nabla^{\perp} B \cdot \nabla u .
$$


Now that we have our equation in the desired divergence-free form, we can show the continuity of the solution $u$ using the Hodge decomposition (see Corollary 10.70 in [7])

$$
\left(i d+\varepsilon^{*}\right) P \nabla u=\nabla V+\nabla^{\perp} W
$$

and arguing as in [15].

\section{Proof of theorem 2.13}

We split the proof of this result into several steps and present each step in a separate subsection.

\subsection{Gauge fixing}

Following the work of de Longueville and Gastel in the proof of Theorem 4.1 (i) in [5] we repeatedly solve Neumann problems to find $\Omega \in W^{m-1,2}\left(B^{2 m}\right.$, so $\left.(n) \otimes \wedge^{1} \mathbb{R}^{2 m}\right)$ such that

$$
\begin{aligned}
& \Delta^{m-2} \delta \Omega=-\eta \quad \text { in } B^{2 m} \text { and } \\
& \|\Omega\|_{W^{m-1,2}\left(B^{2 m}\right)} \leq c\|\eta\|_{W^{2-m, 2}\left(B^{2 m}\right)} \leq c \sigma .
\end{aligned}
$$

Next we need the following higher order version of the Uhlenbeck gauge fixing result which is due to De Longueville and Gastel.

Theorem 4.1 [Theorem 2.4 in [5]] Assume that $m, n \in \mathbb{N}$ and $B_{r} \subset \mathbb{R}^{2 m}$ is a ball of radius $r$. Then there is $\varepsilon>0$ such that for all $\Omega \in W^{m-1,2}\left(B_{r}\right.$, so $\left.(n) \otimes \wedge^{1} \mathbb{R}^{2 m}\right)$ satisfying

$$
\|\Omega\|_{W^{m-1,2}\left(B_{r}\right)}<\varepsilon
$$

there are functions $P \in W^{m, 2}\left(B_{r / 2} ; S O(n)\right)$ and $\xi \in W^{m, 2}\left(B_{r / 2}, \operatorname{so}(n) \otimes \wedge^{2} \mathbb{R}^{2 m}\right)$ such that

$$
\Omega=P d P^{-1}+P \delta \xi P^{-1}
$$

holds on $B_{r / 2}$. Moreover, we have the estimate

$$
\|d P\|_{W^{m-1,2}\left(B_{r / 2}\right)}+\|\delta \xi\|_{W^{m-1,2}\left(B_{r / 2}\right)} \leq c\|\Omega\|_{W^{m-1,2}\left(B_{r}\right)} .
$$

We apply this result for $\sigma>0$ sufficiently small, and get $\xi \in W^{m, 2}\left(B_{1 / 2}^{2 m}, \operatorname{so}(n) \otimes \wedge^{2} \mathbb{R}^{2 m}\right)$ and $P \in W^{m, 2}\left(B_{1 / 2}^{2 m}, S O(n)\right)$ such that

$$
\begin{aligned}
& d P=P \Omega-\delta \xi P \text { and } \\
& \quad\|d P\|_{W^{m-1,2}\left(B_{1 / 2}^{2 m}\right)}+\|\delta \xi\|_{W^{m-1,2}\left(B_{1 / 2}^{2 m}\right)} \leq c\|\Omega\|_{L^{m-1,2}\left(B^{2 m}\right)} .
\end{aligned}
$$




\subsection{Rewriting the system}

We let $\varepsilon \in W^{m, 2} \cap L^{\infty}\left(B_{1 / 2}^{2 m}, M(n)\right)$ and we multiply (2.4) with $(i d+\varepsilon) P$ and calculate

$$
\begin{aligned}
(i d+ & \varepsilon) P \Delta^{m} u=(i d+\varepsilon) P\left[\sum_{k=0}^{m-1} \Delta^{k}\left\langle V_{k}, d u\right\rangle+\sum_{k=0}^{m-2} \Delta^{k} \delta\left(w_{k} d u\right)\right] \\
& \Leftrightarrow\left[\sum_{k=0}^{m-1} \Delta^{k}((i d+\varepsilon) P) V_{k}-\sum_{k=0}^{m-2} d \Delta^{k}((i d+\varepsilon) P) w_{k}+d \Delta^{m-1}((i d+\varepsilon) P)\right] \cdot d u \\
= & \delta\left[\sum_{l=0}^{m-1} \Delta^{l}((i d+\varepsilon) P) \Delta^{m-l-1} d u-\sum_{l=0}^{m-2} d \Delta^{l}((i d+\varepsilon) P) \Delta^{m-l-1} u\right. \\
& -\sum_{k=0}^{m-1} \sum_{l=0}^{k-1} \Delta^{l}((i d+\varepsilon) P) \Delta^{k-l-1} d\left\langle V_{k}, d u\right\rangle \\
& +\sum_{k=0}^{m-1} \sum_{l=0}^{k-1} d \Delta^{l}((i d+\varepsilon) P) \Delta^{k-l-1}\left\langle V_{k}, d u\right\rangle \\
& -\sum_{k=0}^{m-2} \sum_{l=0}^{k} \Delta^{l}((i d+\varepsilon) P) d \Delta^{k-l-1} \delta\left(w_{k} d u\right) \\
& \left.+\sum_{k=0}^{m-2} \sum_{l=0}^{k-1} d \Delta^{l}((i d+\varepsilon) P) \Delta^{k-l-1} \delta\left(w_{k} d u\right)\right]
\end{aligned}
$$

The right-hand side of this system is already in divergence form, hence in order to obtain a conservation law we need to find $\varepsilon \in W^{m, 2} \cap L^{\infty}\left(B_{1 / 2}^{2 m}, M(n)\right)$ such that

$$
\delta\left[\sum_{k=0}^{m-1} \Delta^{k}((i d+\varepsilon) P) V_{k}-\sum_{k=0}^{m-2} d \Delta^{k}((i d+\varepsilon) P) w_{k}+d \Delta^{m-1}((i d+\varepsilon) P)\right]=0
$$

on $B_{1 / 2}^{2 m}$. As in Sect. 3 we want to apply a fixed point argument to solve this problem. However to do this we need to have a certain control on the terms in (4.7) and the terms involving $V_{0}$ are problematic. We know that $V_{0}=d \eta+F$ and we control $F \in W^{2-m, \frac{2 m}{m+1}, 1}\left(B^{2 m}\right)$ by (2.5) but $d \eta \in W^{1-m, 2}\left(B^{2 m}\right)$ is a priori not bounded. Thus our goal is to remove $d \eta$.

To do this we take a closer look at $d \Delta^{m-1}((i d+\varepsilon) P)$ and note that we can rewrite the highest order term $(i d+\varepsilon) d \Delta^{m-1} P$ so that it cancels $(i d+\varepsilon) P d \eta$ in (4.7). To see this we use (2.2), (2.3) as well as (4.1) and (4.5) .

$$
\begin{aligned}
d \Delta^{m-1} P & =d \Delta^{m-2} \delta(P \Omega-\delta \xi P) \\
& =d \Delta^{m-2}(d P \Omega)+d \Delta^{m-2}(P \delta \Omega)-d \Delta^{m-2}(* d *(* d * \xi P)) \\
& =\sum_{i=1}^{2 m-2} c_{i} \nabla^{i} P \nabla^{2 m-2-i} \Omega-d(P \eta)+d \Delta^{m-2}(*(d * \xi \wedge d P)) \\
& =\sum_{i=1}^{2 m-2} c_{i} \nabla^{i} P \nabla^{2 m-2-i} \Omega-d P \eta-P\left(V_{0}-F\right)+d \Delta^{m-2}(*(d * \xi \wedge d P)),
\end{aligned}
$$


with constants $c_{i} \in \mathbb{N}_{0}, 1 \leq i \leq 2 m-2$ and

$$
\nabla^{k}= \begin{cases}\Delta^{\frac{k}{2}}, & \text { if } k \text { even, } \\ d \Delta^{\frac{k-1}{2}}, & \text { if } k \text { odd }\end{cases}
$$

Plugging this back into (4.7) and rearranging we get

$$
\begin{aligned}
\Delta\left(\Delta^{m-1} \varepsilon \cdot P\right)=\delta[ & -\sum_{j=1}^{2 m-2} \tilde{c}_{j} \nabla^{j} \varepsilon \nabla^{2 m-1-j} P-(i d+\varepsilon)\left(\sum_{i=1}^{2 m-2} c_{i} \nabla^{i} P \nabla^{2 m-2-i} \Omega\right. \\
& \left.-d P \eta+P F+d \Delta^{m-2}(*(d * \xi \wedge d P))\right) \\
& \left.-\sum_{k=1}^{m-1} \Delta^{k}((i d+\varepsilon) P) V_{k}+\sum_{k=0}^{m-2} d \Delta^{k}((i d+\varepsilon) P) w_{k}\right] \text { in } B_{1 / 2}^{2 m},
\end{aligned}
$$

where $\tilde{c}_{j}$ are constants in $\mathbb{N}_{0}$. Now that we have removed the "worst" terms we want to examine this equation further and take a closer look at the function spaces of the summands. We separate the $\varepsilon$ component from the rest and use the embedding results for Lorentz-Sobolev spaces in Lemma 2.10 and Lemma 2.11 repeatedly. We use the notation $D^{k} A \star D^{l} B$ for any linear combination of $D^{k} A$ and $D^{l} B$ and $D$ denotes the full derivative. For the first term we have

$$
\sum_{j=1}^{2 m-2} D^{j} \varepsilon \star D^{2 m-1-j} P=\sum_{j=1}^{2 m-2} W^{m-j, 2} \cdot W^{-m+1+j, 2},
$$

For the third and fourth term we get

$$
\begin{aligned}
& (i d+\varepsilon) d P \eta=L^{\infty} \cdot W^{m-1,2} \cdot W^{2-m, 2} \hookrightarrow L^{\infty} \cdot W^{2-m, \frac{2 m}{m+1}, 1}, \\
& (i d+\varepsilon) P F=L^{\infty} \cdot L^{\infty} \cdot W^{2-m, \frac{2 m}{m+1}, 1} .
\end{aligned}
$$

The second term is of the from

$$
\begin{aligned}
(i d & +\varepsilon)\left(\sum_{j=1}^{2 m-3} D^{j} \Omega \star D^{2 m-2-j} P+\Omega \star D^{2 m-2} P\right) \\
= & \sum_{j=1}^{2 m-3} L^{\infty} \cdot W^{m-1-j, 2} \cdot W^{-m+2+j, 2}+L^{\infty} \cdot W^{m-1,2} \cdot W^{2-m, 2} \\
\hookrightarrow & \sum_{j=1}^{m-2} L^{\infty} \cdot W^{-m+2+j, \frac{2 m}{m+1+j}, 1}+\sum_{j=m-1}^{2 m-3} L^{\infty} \cdot W^{m-1-j, \frac{2 m}{3 m-2-j}}, 1 \\
& +L^{\infty} \cdot W^{2-m, \frac{2 m}{m+1}, 1} \\
\hookrightarrow & L^{\infty} \cdot W^{2-m, \frac{2 m}{m+1}, 1},
\end{aligned}
$$

where we used Lemma 2.11 in the first step and Lemma 2.10 with $s=m-2-j, p=$ $\frac{2 m}{m+1+j}, t=j$ for $j=1, \ldots, m-2$ and $s=-m+1+j, p=\frac{2 m}{3 m-2-j}, t=2 m-3-j$ 
for $j=m-1, \ldots, 2 m-3$ in the second step. The fifth term follows in the same way

$$
\begin{aligned}
(i d+\varepsilon) d \Delta^{m-2}(*(d P \wedge d * \xi)) & =(i d+\varepsilon) \sum_{j=1}^{2 m-2} D^{j} \xi \star D^{2 m-1-j} P \\
& =\sum_{j=1}^{2 m-2} L^{\infty} \cdot W^{m-j, 2} \cdot W^{-m+1+j, 2} \\
& \hookrightarrow L^{\infty} \cdot W^{2-m, \frac{2 m}{m+1}, 1} .
\end{aligned}
$$

For the last two terms we apply again Lemma 2.11 and 2.10 with $s=m-2 k-1, p=$ $\frac{2 m}{m+2 k-j}, t=2 k-j$ for $2 k+1-m<m-2 k+j$ and $s=2 k-j-m, p=\frac{2 m}{3 m-2 k-1}, t=$ $2 m-2 k-1$ for $m-2 k+j \leq 2 k+1-m$.

$$
\begin{aligned}
& \sum_{k=1}^{m-1} \Delta^{k}((i d+\varepsilon) P) V_{k}=\sum_{k=1}^{m-1}\left(\sum_{j=1}^{2 k-1} D^{j} \varepsilon \star D^{2 k-j} P+(i d+\varepsilon) \Delta^{k} P+\Delta^{k} \varepsilon P\right) V_{k} \\
& =\sum_{k=1}^{m-1} \sum_{j=1}^{2 k-1} W^{m-j, 2} \cdot W^{m-2 k+j, 2} \cdot W^{2 k+1-m, 2}+\sum_{k=1}^{m-1} L^{\infty} \cdot W^{m-2 k, 2} \cdot W^{2 k+1-m, 2} \\
& \hookrightarrow \sum_{\substack{j, k \in \mathbb{N}, 2 k+1-m<2 k-1, k \leq m-1}} W^{m-j, 2} \cdot W^{2 k+1-m, \frac{2 m}{m+2 k-j}} \\
& +\sum_{\substack{j, k \in \mathbb{N}, j \leq 2 k-1, k \leq m-1 \\
m-2 k+j \leq 2 k+1-m}} W^{m-j, 2} \cdot W^{m-2 k+j, \frac{2 m}{3 m-2 k-1}} \\
& +\sum_{\substack{k \in \mathbb{N}, k \leq m-1 \\
2 k+1-m<m-2 k}} L^{\infty} \cdot W^{2 k+1-m, \frac{2 m}{m+2 k}, 1}+\sum_{\substack{k \in \mathbb{N}, k \leq m-1 \\
m-2 k \leq 2 k+1-m}} L^{\infty} \cdot W^{m-2 k, \frac{2 m}{3 m-2 k-1}, 1} \\
& \hookrightarrow \sum_{j=1}^{2 m-3} W^{m-j, 2} \cdot W^{-m+1+j, 2}+L^{\infty} \cdot W^{2-m, \frac{2 m}{m+1}, 1}
\end{aligned}
$$

and analogously

$$
\begin{aligned}
& \sum_{k=0}^{m-2} \nabla \Delta^{k}((i d+\varepsilon) P) w_{k} \\
& \quad=\sum_{k=0}^{m-2}\left(\sum_{j=1}^{2 k} D^{j} \varepsilon \star D^{2 k+1-j} P+(i d+\varepsilon) \delta \Delta^{k} P+\delta \Delta^{k} \varepsilon P\right) w_{k} \\
& \quad=\sum_{k=0}^{m-2} \sum_{j=1}^{2 k} W^{m-j, 2} \cdot W^{m-2 k-1+j, 2} \cdot W^{2 k+2-m, 2}+\sum_{k=0}^{m-2} L^{\infty} \cdot W^{m-2 k+1} \cdot W^{2 k+2-m, 2} \\
& \hookrightarrow \sum_{j=1}^{2 m-3} W^{m-j, 2} \cdot W^{-m+1+j, 2}+L^{\infty} \cdot W^{2-m, \frac{2 m}{m+1}, 1} \cdot
\end{aligned}
$$

Observe that all terms on the right-hand side of (4.8) consist of products $W^{m-j, 2}$. $W^{j+1-m, 2}, j=1, \ldots, 2 m-2$ and $L^{\infty} \cdot W^{2-m, \frac{2 m}{m+1}, 1}$. Thus we can simplify (4.8) further 
and write

$$
\Delta\left(\Delta^{m-1} \varepsilon \cdot P\right)=\delta\left(\sum_{j=1}^{2 m-2} D^{j} \varepsilon \star K_{j}+(i d+\varepsilon) \star K_{0}\right)
$$

with $K_{j} \in W^{j+1-m, 2}\left(B_{1 / 2}^{2 m}\right), K_{0} \in W^{2-m, \frac{2 m}{m+1}, 1}\left(B_{1 / 2}^{2 m}\right)$. Moreover with (4.4) and (2.5) we estimate

$$
\left\|K_{0}\right\|_{W^{2-m, \frac{2 m}{m+1}, 1}\left(B_{1 / 2}^{2 m}\right)}+\sum_{j=1}^{2 m-2}\left\|K_{j}\right\|_{W^{j+1-m, 2}\left(B_{1 / 2}^{2 m}\right)} \leq c \sigma .
$$

However the equation still contains distributions. To take care of these we apply the same technique as de Longueville and Gastel and use the representation of negative LorentzSobolev spaces (see Lemma 2.8).

$$
\begin{array}{rlrl}
\varepsilon & =\sum_{|\alpha| \leq m-2} \partial^{\alpha} \varepsilon_{\alpha}, & \varepsilon_{\alpha} \in W^{2 m-1, \frac{2 m}{2 m-1-|\alpha|}, 1}\left(B_{1 / 2}^{2 m}\right), \\
K_{0} & =\sum_{|\alpha| \leq m-2} \partial^{\alpha} K_{0}^{\alpha}, & & K_{0}^{\alpha} \in L^{\frac{2 m}{m+1}, 1}\left(B_{1 / 2}^{2 m}\right), \\
K_{j} & =\sum_{|\alpha| \leq m-1-j} \partial^{\alpha} K_{j}^{\alpha}, & & K_{j}^{\alpha} \in L^{2}\left(B_{1 / 2}^{2 m}\right) .
\end{array}
$$

Together with (4.10) we get

$$
\begin{aligned}
& \sum_{|\alpha| \leq m-1-j}\left\|K_{j}^{\alpha}\right\|_{L^{2}\left(B_{1 / 2}^{2 m}\right)} \leq c\left\|K_{j}\right\|_{W^{j+1-m, 2}\left(B_{1 / 2}^{2 m}\right)} \leq c \sigma \\
& \sum_{|\alpha| \leq m-2}\left\|K_{0}^{\alpha}\right\|_{L^{\frac{2 m}{m+1}, 1}\left(B_{1 / 2}^{2 m}\right)} \leq c\left\|K_{0}\right\|_{W^{2-m, \frac{2 m}{m+1}, 1}\left(B_{1 / 2}^{2 m}\right)} \leq c \sigma .
\end{aligned}
$$

Note that we assume $\varepsilon \in W^{m+1, \frac{2 m}{m+1}, 1}$ for this representation, which is slightly better than the original assumption $\varepsilon \in W^{m, 2} \cap L^{\infty}$. We will see that we can solve (4.8) in this better space and since $W^{m+1, \frac{2 m}{m+1}, 1}\left(B^{2 m}\right) \hookrightarrow W^{m, 2} \cap L^{\infty}\left(B^{2 m}\right)$ we get the desired result.

This new representation allows us to shift derivatives away from the distributional part. Let $c_{\alpha \gamma}, c_{\beta \gamma} \in \mathbb{Z}$. With the product rule we get for $j=1, \ldots, m-2$

$$
D^{j} \varepsilon \star K_{j}=\sum_{\substack{|\alpha| \leq m-2 \\|\beta| \leq m-1-j}} D^{j} \partial^{\alpha} \varepsilon_{\alpha} \star \partial^{\beta} K_{j}^{\beta}=\sum_{\substack{|\alpha| \leq m-2 \\|\beta| \leq m-1-j}} \sum_{\gamma \leq \beta} \partial^{\gamma}\left(c_{\beta \gamma} \partial^{\beta-\gamma} \partial^{\alpha} D^{j} \varepsilon_{\alpha} \star K_{j}^{\beta}\right)
$$

The case $j=0$ follows analogously

$$
\begin{aligned}
(i d+\varepsilon) \star K_{0} & =\sum_{|\gamma| \leq m-2} \partial^{\gamma} K_{0}^{\gamma}+\sum_{\substack{|\alpha| \leq m-2 \\
|\beta| \leq m-2}} \partial^{\alpha} \varepsilon_{\alpha} \star \partial^{\beta} K_{0}^{\beta} \\
& =\sum_{|\gamma| \leq m-2} \partial^{\gamma} K_{0}^{\gamma}+\sum_{\substack{|\alpha| \leq m-2 \\
|\beta| \leq m-2}} \sum_{\gamma \leq \beta} \partial^{\gamma}\left(c_{\beta \gamma} \partial^{\beta-\gamma} \partial^{\alpha} \varepsilon_{\alpha} \star K_{0}^{\beta}\right) .
\end{aligned}
$$

For $j=m-1, \ldots, 2 m-2$ with $|\alpha| \leq j+1-m$ we get

$$
D^{j} \varepsilon \star K_{j}=\sum_{|\alpha| \leq m-2} D^{j} \partial^{\alpha} \varepsilon_{\alpha} \star K_{j}=\sum_{|\alpha| \leq m-2} \sum_{\gamma \leq \alpha} \partial^{\gamma}\left(c_{\alpha \gamma} D^{j} \varepsilon_{\alpha} \star \partial^{\alpha-\gamma} K_{j}\right) \text {. }
$$


If $|\alpha|>j+1-m$ we choose $\beta \leq \alpha$ with $|\beta|=j+1-m$ and

$$
\begin{aligned}
D^{j} \varepsilon \star K_{j} & =\sum_{|\alpha| \leq m-2} D^{j} \partial^{\alpha} \varepsilon_{\alpha} \star K_{j} \\
& =\sum_{|\alpha| \leq m-2} \sum_{\substack{\gamma \leq \beta \\
|\beta|=j+1-m}} \partial^{\gamma}\left(c_{\beta \gamma} \partial^{\alpha-\beta} D^{j} \varepsilon_{\alpha} \star \partial^{\beta-\gamma} K_{j}\right) .
\end{aligned}
$$

We rewrite the left-hand side of (4.9) in the same way.

$$
\begin{aligned}
\Delta\left(\Delta^{m-1} \varepsilon \cdot P\right) & =\sum_{|\alpha| \leq m-2} \Delta\left(\Delta^{m-1} \partial^{\alpha} \varepsilon_{\alpha} \cdot P\right) \\
& =\sum_{|\alpha| \leq m-2} \sum_{\gamma \leq \alpha} \partial^{\gamma} \Delta\left(c_{\alpha \gamma} \Delta^{m-1} \varepsilon_{\alpha} \partial^{\alpha-\gamma} P\right) \\
& =\sum_{|\gamma| \leq m-2} \partial^{\gamma} \Delta\left(\Delta^{m-1} \varepsilon_{\gamma} \cdot P\right)+\sum_{|\alpha| \leq m-2} \sum_{\gamma<\alpha} \partial^{\gamma} \Delta\left(c_{\alpha \gamma} \Delta^{m-1} \varepsilon_{\alpha} \partial^{\alpha-\gamma} P\right) .
\end{aligned}
$$

For the last term note that $P \in W^{m, 2}\left(B_{1 / 2}^{2 m}, S O(n)\right)$. Thus we identify $P$ with $K_{2 m-1}$ and write

$$
\begin{aligned}
& \sum_{|\alpha| \leq m-2} \sum_{\gamma<\alpha} \partial^{\gamma} \Delta\left(c_{\alpha \gamma} \Delta^{m-1} \varepsilon_{\alpha} \partial^{\alpha-\gamma} P\right) \\
& =\delta\left[\sum_{|\alpha| \leq m-2} \sum_{\gamma<\alpha} \sum_{i=0}^{1} \partial^{\gamma}\left(c_{\alpha \gamma} D^{2 m-2-i} \varepsilon_{\alpha} \star \partial^{\alpha-\gamma} D^{1-i} K_{2 m-1}\right)\right] .
\end{aligned}
$$

Putting all of this together we get an equation equivalent to (4.7)

$$
\begin{aligned}
& \sum_{|\gamma| \leq m-2} \partial^{\gamma} \Delta\left(\Delta^{m-1} \varepsilon_{\gamma} \cdot P\right) \\
& =\delta\left[\sum_{|\gamma| \leq m-2} \partial^{\gamma} K_{0}^{\gamma}+\sum_{\substack{|\alpha| \leq m-2 \\
|\beta| \leq m-2}} \sum_{\gamma \leq \beta} \partial^{\gamma}\left(c_{\beta \gamma} \partial^{\beta-\gamma} \partial^{\alpha} \varepsilon_{\alpha} \star K_{0}^{\beta}\right)\right. \\
& +\sum_{j=1}^{m-2} \sum_{\substack{|\alpha| \leq m-2 \\
|\beta| \leq m-1-j}} \sum_{\gamma \leq \beta} \partial^{\gamma}\left(c_{\beta \gamma} \partial^{\beta-\gamma} \partial^{\alpha} D^{j} \varepsilon_{\alpha} \star K_{j}^{\beta}\right) \\
& +\sum_{\substack{j=m-1 \\
|\alpha| \leq j+1-m}}^{2 m-2} \sum_{|\alpha| \leq m-2} \sum_{\gamma \leq \alpha} \partial^{\gamma}\left(c_{\alpha \gamma} D^{j} \varepsilon_{\alpha} \star \partial^{\alpha-\gamma} K_{j}\right) \\
& +\sum_{\substack{j=m-1 \\
|\alpha|>j+1-m}}^{2 m-2} \sum_{|\alpha| \leq m-2} \sum_{\substack{\gamma \leq \beta \\
|\beta|=j+1-m}} \partial^{\gamma}\left(c_{\beta \gamma} \partial^{\alpha-\beta} D^{j} \varepsilon_{\alpha} \star \partial^{\beta-\gamma} K_{j}\right) \\
& \left.+\sum_{i=0}^{1} \sum_{|\alpha| \leq m-2} \sum_{\gamma<\alpha} \partial^{\gamma}\left(c_{\alpha \gamma} D^{2 m-2-i} \varepsilon_{\alpha} \star \partial^{\alpha-\gamma} D^{1-i} K_{2 m-1}\right)\right] \text {. }
\end{aligned}
$$


We simplify this further by setting

$$
\sum_{|\gamma| \leq m-2} \partial^{\gamma} \Delta\left(\Delta^{m-1} \varepsilon_{\gamma} \cdot P\right)=: \delta\left[\sum_{|\gamma| \leq m-2} \partial^{\gamma}\left(\langle\varepsilon, K\rangle_{\gamma}+K_{0}^{\gamma}\right)\right]
$$

with

$$
\begin{aligned}
& \left\|K_{0}^{\gamma}\right\|_{L^{\frac{2 m}{2 m-1-|\gamma|}, 1}\left(B_{1 / 2}^{2 m}\right)}+\left\|\langle\varepsilon, K\rangle_{\gamma}\right\|_{L^{\frac{2 m}{2 m-1-|\gamma|}, 1}\left(B_{1 / 2}^{2 m}\right)} \\
& \leq c \sigma\left(\sum_{|\alpha| \leq m-2}\left\|\varepsilon_{\alpha}\right\|_{W^{2 m-1, \frac{2 m}{2 m-1-|\alpha|}, 1}\left(B_{1 / 2}^{2 m}\right)}+1\right)
\end{aligned}
$$

for every $\gamma$ with $|\gamma| \leq m-2$. To see this last inequality we use (4.12) and estimate each term separately

$$
\left\|K_{0}^{\gamma}\right\|_{L^{\frac{2 m}{2 m-1-|\gamma|}, 1}\left(B_{1 / 2}^{2 m}\right)} \leq c\left\|K_{0}^{\gamma}\right\|_{L^{\frac{2 m}{m+1}, 1}\left(B_{1 / 2}^{2 m}\right)} \leq c \sigma
$$

since $K_{0}^{\gamma} \in L^{\frac{2 m}{m+1}, 1}$ and $L^{\frac{2 m}{m+1}, 1} \hookrightarrow L^{\frac{2 m}{2 m-1-|\gamma|}, 1}\left(B_{1 / 2}^{2 m}\right)$ by Lemma 2.2. Further we have

$$
W^{2 m-1-|\beta|+|\gamma|-|\alpha|-j, \frac{2 m}{2 m-1-|\alpha|}, 1} \hookrightarrow L^{\frac{2 m}{j+|\beta|-|\gamma|}, 1} \hookrightarrow L^{\frac{2 m}{m-1-|\gamma|}, 1}\left(B_{1 / 2}^{2 m}\right)
$$

by Lemma 2.4 and Lemma 2.2 since $|\beta| \leq m-j-1$. With Lemma 2.1 and 2.2 we have $L^{\frac{2 m}{m-1-|\gamma|}, 1} \cdot L^{2} \hookrightarrow L^{\frac{2 m}{2 m-1-|\gamma|}, 1}$ and since $\gamma \leq \beta$

$$
\begin{aligned}
& \left\|\partial^{\beta-\gamma} \partial^{\alpha} D^{j} \varepsilon_{\alpha} \star K_{j}^{\beta}\right\|_{L^{\frac{2 m}{2 m-1-|\gamma|}, 1}\left(B_{1 / 2}^{2 m}\right)} \\
& \quad \leq c\left\|\varepsilon_{\alpha}\right\|_{W^{2 m-1-j-|\alpha|-|\beta|+|\gamma|, \frac{2 m}{2 m-1-|\alpha|}, 1}\left(B_{1 / 2}^{2 m}\right)}\left\|K_{j}^{\beta}\right\|_{L^{2}\left(B_{1 / 2}^{2 m}\right)} \\
& \quad \leq c \sigma\left\|\varepsilon_{\alpha}\right\|_{W^{2 m-1, \frac{2 m}{2 m-1-|\alpha|}, 1}\left(B_{1 / 2}^{2 m}\right)} .
\end{aligned}
$$

The remaining terms follow in a similar way. With Lemma 2.4

$$
W^{2 m-1-|\beta|+|\gamma|-|\alpha|, \frac{2 m}{2 m-1-|\alpha|}, 1} \hookrightarrow L^{\frac{2 m}{|\beta|-|\gamma|}, 1}\left(B_{1 / 2}^{2 m}\right)
$$

and by Lemma 2.1 and 2.2 with $|\beta| \leq m-2$

$$
L^{\frac{2 m}{|\beta|-|\gamma|}, 1} \cdot L^{\frac{2 m}{m+1}, 1} \hookrightarrow L^{\frac{2 m}{m+|\beta|-|\gamma|+1}, 1} \hookrightarrow L^{\frac{2 m}{2 m-1-|\gamma|}, 1}\left(B_{1 / 2}^{2 m}\right) .
$$

With this and $\gamma \leq \beta$

$$
\begin{aligned}
& \left\|\partial^{\beta-\gamma} \partial^{\alpha} \varepsilon_{\alpha} \star K_{0}^{\beta}\right\|_{L^{2 m-1-|\gamma|}, 1}
\end{aligned}
$$

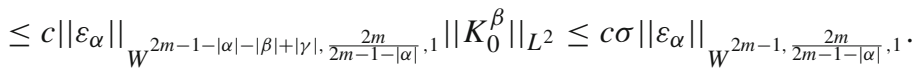

For the next term we have with Lemma 2.4 and 2.1

$$
\begin{aligned}
W^{2 m-1-j, \frac{2 m}{2 m-1-|\alpha|}, 1} \cdot W^{j+1-m-|\alpha|+|\gamma|, 2} & \hookrightarrow L^{\frac{2 m}{j-|\alpha|}, 1} \cdot L^{\frac{2 m}{2 m+|\alpha|-|\gamma|-j-1}, 2} \\
& \hookrightarrow L^{\frac{2 m}{2 m-1-|\gamma|}, 1}\left(B_{1 / 2}^{2 m}\right)
\end{aligned}
$$


so that with $\gamma \leq \alpha$

$$
\begin{aligned}
& \left\|D^{j} \varepsilon_{\alpha} \star \partial^{\alpha-\gamma} K_{j}\right\|_{L^{\frac{2 m}{2 m-1-|\gamma|}, 1}}
\end{aligned}
$$

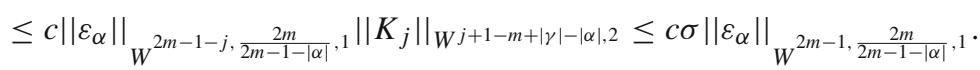

In the fifth term we use $|\beta|=j+1-m$, Lemma 2.4 and 2.1 to get

$$
\begin{aligned}
W^{2 m-1-|\alpha|+|\beta|-j, \frac{2 m}{2 m-1-|\alpha|}, 1} \cdot W^{j+1-m-|\beta|+|\gamma|, 2} & \hookrightarrow L^{\frac{2 m}{m-1}, 1} \cdot L^{\frac{2 m}{m-|\gamma|}, 2} \\
& \hookrightarrow L^{\frac{2 m}{2 m-1-|\gamma|}, 1}\left(B_{1 / 2}^{2 m}\right)
\end{aligned}
$$

and

$$
\begin{aligned}
& \left\|\partial^{\alpha-\beta} D^{j} \varepsilon_{\alpha} \star \partial^{\beta-\gamma} K_{j}\right\|_{L^{\frac{2 m}{2 m-1-|\gamma|}}, 1} \\
& \quad \leq c\left\|\varepsilon_{\alpha}\right\|_{W^{2 m-1-j-|\alpha|+|\beta|, \frac{2 m}{2 m-1-|\alpha|}, 1} \mid}\left\|K_{j}\right\|_{W^{j+1-m+|\gamma|-|\beta|, 2}} \\
& \quad \leq c \sigma\left\|\varepsilon_{\alpha}\right\|_{W^{2 m-1, \frac{2 m}{2 m-1-|\alpha|}, 1} .} .
\end{aligned}
$$

Finally we estimate for $i=0,1$ with (4.4) and $\gamma \leq \alpha$

$$
\begin{aligned}
& \left\|D^{2 m-2-i} \varepsilon_{\alpha} \star \partial^{\alpha-\gamma} D^{1-i} K_{2 m-1}\right\|_{L^{\frac{2 m}{2 m-1-|\gamma|}, 1}} \\
& \quad \leq\left\|\varepsilon_{\alpha}\right\|_{W^{1+i, \frac{2 m}{2 m-1-|\alpha|}, 1}}\|P\|_{W^{m-|\alpha|+|\gamma|-1+i, 2}} \leq c \sigma\left\|\varepsilon_{\alpha}\right\|_{W^{2 m-1, \frac{2 m}{2 m-1-|\alpha|}, 1}}
\end{aligned}
$$

and this proves (4.14).

\subsection{The fixed point argument}

Instead of solving (4.13) we solve the system

$$
\Delta\left(\Delta^{m-1} \varepsilon_{\gamma} \cdot P\right)=\delta\left(\langle\varepsilon, K\rangle_{\gamma}+K_{0}^{\gamma}\right) \quad \text { for every } \gamma \text { with }|\gamma| \leq m-2 .
$$

To do this we apply a fixed point argument: Let $X_{\gamma}:=\left\{u \in M(n):\|u\|_{W^{2 m-1, \frac{2 m}{2 m-1-|\gamma|}, 1}}\left(B_{1 / 2}^{2 m}\right)\right.$ $<\infty\}$ and $X=\oplus_{|\gamma| \leq m-2} X_{\gamma}$. We define maps $\psi_{\gamma}: X_{\gamma} \rightarrow X_{\gamma}$ by

$$
\psi_{\gamma}: \varepsilon_{\gamma} \mapsto \text { solution } \lambda_{\gamma} \text { of (4.16) }
$$

with

$$
\left\{\begin{array}{rlrl}
\Delta\left(\Delta^{m-1} \lambda_{\gamma} \cdot P\right) & =\delta\left(\langle\varepsilon, K\rangle_{\gamma}+K_{0}^{\gamma}\right) & & \text { in } B_{1 / 2}^{2 m}, \\
\Delta^{j} \lambda_{\gamma} & =0 & \text { on } \partial B_{1 / 2}^{2 m} \text { for } j=0, \ldots, m-1 .
\end{array}\right.
$$

Let $\hat{\lambda}=\sum_{|\gamma| \leq m-2} \lambda_{\gamma}$ and $\hat{\varepsilon}=\sum_{|\gamma| \leq m-2} \varepsilon_{\gamma}$, where $\lambda_{\gamma}$ is a solution of (4.16) for every $\gamma$ with corresponding $\varepsilon_{\gamma}$. Let $\Psi=\bigoplus_{|\gamma| \leq m-2} \psi_{\gamma}$ and

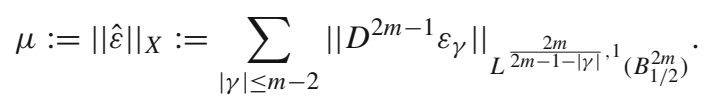


We apply Lemma 2.12 and (4.14) to estimate

$$
\begin{aligned}
\left\|D^{2 m-1} \lambda_{\gamma}\right\|_{L^{\frac{2 m}{2 m-1-|\gamma|}, 1}\left(B_{1 / 2}^{2 m}\right)} & \leq c\left\|\langle\varepsilon, K\rangle_{\gamma}+K_{0}^{\gamma}\right\|_{L^{\frac{2 m}{2 m-1-|\gamma|}, 1}\left(B_{1 / 2}^{2 m}\right)} \\
& \leq c \sigma\left(\sum_{|\gamma| \leq m-2}\left\|\varepsilon_{\gamma}\right\|_{W^{2 m-1, \frac{2 m}{2 m-1-|\gamma|}, 1}\left(B_{1 / 2}^{2 m}\right)}+1\right) \\
& \leq c_{1} \sigma(\mu+1) .
\end{aligned}
$$

We choose $\sigma<\frac{\mu}{2 c_{1}(\mu+1)}$ to get

$$
\|\hat{\lambda}\|_{X} \leq \frac{\mu}{2}
$$

Next we show that $\psi_{\gamma}$ is a contraction. Let $\lambda_{\gamma}^{1}, \lambda_{\gamma}^{2}$ be solutions of (4.16) with $\varepsilon_{\gamma}^{1}, \varepsilon_{\gamma}^{2}$ respectively. Then $\Lambda_{\gamma}:=\lambda_{\gamma}^{1}-\lambda_{\gamma}^{2}$ is a solution of

$$
\left\{\begin{aligned}
\Delta\left(\Delta^{m-1} \Lambda_{\gamma} \cdot P\right) & =\delta\left(\left\langle\varepsilon^{1}-\varepsilon^{2}, K\right\rangle_{\gamma}\right) & & \text { in } B_{1 / 2}^{2 m}, \\
\Delta^{j} \Lambda_{\gamma} & =0 & & \text { on } \partial B_{1 / 2}^{2 m} \text { for } j=0, \ldots, m-1 .
\end{aligned}\right.
$$

Applying Lemma 2.12 and (4.14) again yields

$$
\begin{aligned}
& \left\|D^{2 m-1} \lambda_{\gamma}^{1}-D^{2 m-1} \lambda_{\gamma}^{2}\right\|_{L^{\frac{2 m}{2 m-1-|\gamma|}, 1}\left(B_{1 / 2}^{2 m}\right)} \\
& \leq c \sigma \sum_{|\gamma| \leq m-2}\left\|\varepsilon_{\gamma}^{1}-\varepsilon_{\gamma}^{2}\right\|_{W^{2 m-1, \frac{2 m}{2 m-1-|\gamma|}, 1}\left(B_{1 / 2}^{2 m}\right)} .
\end{aligned}
$$

With this we have

$$
\left\|\hat{\lambda}^{1}-\hat{\lambda}^{2}\right\|_{X} \leq c_{2} \sigma\left\|\hat{\varepsilon}^{1}-\hat{\varepsilon}^{2}\right\|_{X}
$$

Choosing $\sigma<\min \left\{\frac{\mu}{2 c_{1}(\mu+1)}, \frac{1}{2 c_{2}}\right\}$ shows that $\Psi$ is a contraction. Now we can apply the Banach fixed point theorem which yields a unique $\hat{\varepsilon}^{*} \in X$ solving (4.15) and by Lemma 2.12 and (4.14)

$$
\sum_{|\gamma| \leq m-2}\left\|\varepsilon_{\gamma}^{*}\right\|_{W^{2 m-1, \frac{2 m}{2 m-1-|\gamma|}, 1}\left(B_{1 / 2}^{2 m}\right)} \leq c \sigma .
$$

Thus we have

$$
0=\delta\left(d \Delta^{m-1} \varepsilon_{\gamma}^{*} \cdot P-\left\langle\varepsilon^{*}, K\right\rangle_{\gamma}+K_{0}^{\gamma}\right)
$$

for every $\gamma$ with $|\gamma| \leq m-2$. What is left to show is that these $\varepsilon_{\gamma}^{*}$ are the Sobolev functions in the representation (4.11) of $\varepsilon$ and this $\varepsilon$ solves (4.7).

\subsection{Going back to the original system}

In order to go back to our original system, we reverse the abbreviations we made at the beginning to get a detailed look at (4.17). To do this we go back to (4.8). As we have seen before, each term of this equation is a product of a distribution and a Sobolev function. More precisely, the terms are of the form $L^{\infty} \cdot W^{2-m, \frac{2 m}{m-1}}$ and $W^{m-k, 2} \cdot W^{-m+1+k, 2}, k=$ 
$1, \ldots, 2 m-2$. We use the following representations for the distributions according to Lemma 2.8

$$
\begin{aligned}
& F P-d \Delta^{m-2} \delta(\Omega P)+d \Delta^{m-2} \delta \Omega P-d \Delta^{m-2}(*(d P \wedge d * \xi)) \\
& =\sum_{|\alpha| \leq m-2}\left(F P-d \Delta^{m-2} \delta(\Omega P)+d \Delta^{m-2} \delta \Omega P-d \Delta^{m-2}(*(d P \wedge d * \xi))\right)^{\alpha}, \\
& \left(F P-d \Delta^{m-2} \delta(\Omega P)+d \Delta^{m-2} \delta \Omega P-d \Delta^{m-2}(*(d P \wedge d * \xi))\right)^{\alpha} \in L^{\frac{2 m}{m+1}, 1}\left(B_{1 / 2}^{2 m}\right) \\
& \Delta^{k} P \cdot V_{k}=\sum_{|\alpha| \leq m-2} \partial^{\alpha}\left(\Delta^{k} P V_{k}\right)^{\alpha}, \quad\left(\Delta^{k} P V_{k}\right)^{\alpha} \in L^{\frac{2 m}{m+1}, 1}\left(B_{1 / 2}^{2 m}\right), k \neq 0 \\
& d \Delta^{k} P w_{k}=\sum_{|\alpha| \leq m-2} \partial^{\alpha}\left(d \Delta^{k} P w_{k}\right)^{\alpha}, \quad\left(d \Delta^{k} P w_{k}\right)^{\alpha} \in L^{\frac{2 m}{m+1}, 1}\left(B_{1 / 2}^{2 m}\right) \\
& \nabla^{2 k-l} P \cdot V_{k}=\sum_{|\alpha| \leq m-1-l} \partial^{\alpha}\left(\nabla^{2 k-l} P V_{k}\right)^{\alpha}, \quad\left(\nabla^{2 k-l} P V_{k}\right)^{\alpha} \in L^{2}\left(B_{1 / 2}^{2 m}\right), k \neq 0 \\
& \nabla^{2 k+1-l} P \cdot w_{k}=\sum_{|\alpha| \leq m-1-l} \partial^{\alpha}\left(\nabla^{2 k+1-l} P w_{k}\right)^{\alpha}, \quad\left(\nabla^{2 k+1-l} P w_{k}\right)^{\alpha} \in L^{2}\left(B_{1 / 2}^{2 m}\right), \\
& \nabla^{2 m-1-k} P=\sum_{|\alpha| \leq m-1-k} \partial^{\alpha}\left(\nabla^{2 m-1-k} P\right)^{\alpha}, \quad\left(\nabla^{2 m-1-k} P\right)^{\alpha} \in L^{2}\left(B_{1 / 2}^{2 m}\right) .
\end{aligned}
$$

Then we shift derivatives to get an equation of the form $\sum_{|\gamma| \leq m-2} \partial^{\gamma}(\ldots)_{\gamma}=0$ as in (4.13). Using this we see that (4.17) is equivalent to

$$
\begin{aligned}
0= & \delta\left[\sum_{\substack{1 \leq k \leq m-2 \\
|\alpha| \leq m-1-k}} c_{k, \alpha \gamma} \partial^{\alpha-\gamma} \nabla^{k} \partial^{\beta} \varepsilon_{\beta}^{*}\left(\nabla^{2 m-1-k} P\right)^{\alpha}\right. \\
& +\sum_{\substack{m-1 \leq k \leq 2 m-1 \\
|\alpha| \leq k+1-m}} c_{k, \alpha \gamma} \nabla^{k} \varepsilon_{\alpha}^{*} \partial^{\alpha-\gamma} \nabla^{2 m-1-k} P \\
& +\sum_{\substack{m-1 \leq k \leq 2 m-1 \\
|\alpha|>m-1-k \\
|\beta|=m-1-k}} c_{k, \beta \gamma} \partial^{\alpha-\beta} \nabla^{k} \varepsilon_{\alpha}^{*} \partial^{\beta-\gamma} \nabla^{2 m-1-k} P \\
& +\left(F P-d \Delta^{m-2} \delta(\Omega P)+d \Delta^{m-2} \delta \Omega P-d \Delta^{m-2}(*(d P \wedge d * \xi))\right)^{\gamma} \\
& +\sum_{|\alpha|,|\beta| \leq m-2} c_{\beta \gamma} \partial^{\beta-\gamma} \partial^{\alpha} \varepsilon_{\alpha}^{*}\left(F P-d \Delta^{m-2} \delta(\Omega P)+d \Delta^{m-2} \delta \Omega P\right. \\
& \left.-d \Delta^{m-2}(*(d P \wedge d * \xi))\right)^{\beta} \\
& +\sum_{k=1}^{m-1}\left(\Delta^{k} P V_{k}\right)^{\gamma}+\sum_{k=0}^{m-1} \sum_{|\alpha|,|\beta| \leq m-2} c_{\beta \gamma} \partial^{\beta-\gamma} \partial^{\alpha} \varepsilon_{\alpha}^{*}\left(\Delta^{k} P V_{k}\right)^{\beta} \\
& +\sum_{k=1}^{m-1} \sum_{\substack{1 \leq l \leq m-2 \\
l \leq 2 k}} c_{l, \alpha \gamma} \partial^{\alpha-\gamma} \nabla^{l} \partial^{\beta} \varepsilon_{\beta}^{*}\left(\nabla^{2 k-l} P V_{k}\right)^{\alpha}
\end{aligned}
$$




$$
\begin{aligned}
& +\sum_{k=1}^{m-1} \sum_{\substack{m-1 \leq l \leq 2 m-2 \\
l \leq 2 k \\
|\alpha| \leq l+1-m}} c_{l, \alpha \gamma} \nabla^{l} \varepsilon_{\alpha}^{*} \partial^{\alpha-\gamma} \nabla^{2 k-l} P V_{k} \\
& +\sum_{k=1}^{m-1} \sum_{\substack{m-1 \leq l \leq 2 m-2 \\
l \leq 2 k \\
|\alpha|>l+1-m \\
|\beta|=l+1-m}} c_{l, \beta \gamma} \partial^{\alpha-\beta} \nabla^{l} \varepsilon_{\alpha}^{*} \partial^{\beta-\gamma} \nabla^{2 k-l} P V_{k} \\
& -\sum_{k=0}^{m-2}\left(d \Delta^{k} P w_{k}\right)^{\gamma}-\sum_{k=0}^{m-2} \sum_{|\alpha|,|\beta| \leq m-2} c_{\beta \gamma} \partial^{\beta-\gamma} \partial^{\alpha} \varepsilon_{\alpha}^{*}\left(d \Delta^{k} P w_{k}\right)^{\beta} \\
& -\sum_{k=0}^{m-2} \sum_{\substack{1 \leq l \leq m-2 \\
l \leq 2 k+1 \\
|\alpha| \leq l+1-m}} c_{l, \alpha \gamma} \partial^{\alpha-\gamma} \nabla^{l} \partial^{\beta} \varepsilon_{\beta}^{*}\left(\nabla^{2 k+1-l} P w_{k}\right)^{\alpha} \\
& -\sum_{k=0}^{m-2} \sum_{\substack{m-1 \leq l \leq 2 m-3 \\
l \leq 2 k+1 \\
|\alpha| \leq l+1-m}} c_{l, \alpha \gamma} \nabla^{l} \varepsilon_{\alpha}^{*} \partial^{\alpha-\gamma} \nabla^{2 k+1-l} P w_{k} \\
& \left.-\sum_{k=0}^{m-2} \sum_{\begin{array}{c}
m-1 \leq l \leq 2 m-3 \\
l \leq 2 k+1 \\
|\alpha|>l+1-m \\
\beta \mid=l+1-m
\end{array}} c_{l, \beta \gamma} \partial^{\alpha-\beta} \nabla^{l} \varepsilon_{\alpha}^{*} \partial^{\beta-\gamma} \nabla^{2 k+1-l} P w_{k}\right] \\
& =: \delta[\ldots]_{\gamma} \text {. }
\end{aligned}
$$

By the Poincaré Lemma (see Lemma 10.68 in [7]) there exist $B_{\gamma} \in W_{\text {loc }}^{1, \frac{2 m}{2 m-2-|\gamma|}}\left(B_{1 / 2}^{2 m}, \mathbb{R}^{n \times n} \otimes\right.$ $\left.\wedge^{2} \mathbb{R}^{2 m}\right)$ for $|\gamma| \leq m-2$ such that

$$
\delta B_{\gamma}=[\ldots]_{\gamma}
$$

Now we transform $\hat{\varepsilon}^{*}=\sum_{|\gamma| \leq m-2} \varepsilon_{\gamma}^{*}$ and $\hat{B}=\sum_{|\gamma| \leq m-2} B_{\gamma}$ back. Then we have $\varepsilon \in$ $W^{m+1, \frac{2 m}{m-1}, 1}\left(B_{1 / 2}^{2 m}, M(n)\right)$ with

$$
\|\varepsilon\|_{W^{m+1, \frac{2 m}{m-1}, 1}\left(B_{1 / 2}^{2 m}\right)}+\|\varepsilon\|_{L^{\infty}\left(B_{1 / 2}^{2 m}\right)} \leq c \sigma
$$

and

$$
\varepsilon=\sum_{|\gamma| \leq m-2} \partial^{\gamma} \varepsilon_{\gamma}^{*} \quad \text { solves (4.7). }
$$

Further $B=\sum_{|\gamma| \leq m-2} \partial^{\gamma} B_{\gamma} \in W_{\text {loc }}^{2-m, 2}\left(B_{1 / 2}^{2 m}, \mathbb{R}^{n \times n} \otimes \wedge^{2} \mathbb{R}^{2 m}\right)$ with

$$
\delta B=\sum_{k=0}^{m-1} \Delta^{k}((i d+\varepsilon) P) V_{k}-\sum_{k=0}^{m-2} d \Delta^{k}((i d+\varepsilon) P) w_{k}+d \Delta^{m-1}((i d+\varepsilon) P)
$$


and

$$
\begin{aligned}
& \delta\left[\sum_{l=0}^{m-1} \Delta^{l}((i d+\varepsilon) P) \Delta^{m-l-1} d u-\sum_{l=0}^{m-2} d \Delta^{l}((i d+\varepsilon) P) \Delta^{m-l-1} u\right. \\
& \quad-\sum_{k=0}^{m-1} \sum_{l=0}^{k-1} \Delta^{l}((i d+\varepsilon) P) \Delta^{k-l-1} d\left\langle V_{k}, d u\right\rangle \\
& \quad+\sum_{k=0}^{m-1} \sum_{l=0}^{k-1} d \Delta^{l}((i d+\varepsilon) P) \Delta^{k-l-1}\left\langle V_{k}, d u\right\rangle \\
& \quad-\sum_{k=0}^{m-2} \sum_{l=0}^{k} \Delta^{l}((i d+\varepsilon) P) d \Delta^{k-l-1} \delta\left(w_{k} d u\right) \\
& \left.\quad+\sum_{k=0}^{m-2} \sum_{l=0}^{k-1} d \Delta^{l}((i d+\varepsilon) P) \Delta^{k-l-1} \delta\left(w_{k} d u\right)-\langle B, d u\rangle\right]=0 .
\end{aligned}
$$

\subsection{Regularity}

To show ( ii i) we abbreviate the conservation law (2.6)

$$
\Delta\left((i d+\varepsilon) P \Delta^{m-1} u\right)+\delta C=0 \quad \text { on } B_{1 / 2}^{2 m},
$$

where $C \in W^{2-m, \frac{2 m}{m+1}, 1}\left(B_{1 / 2}^{2 m}\right)$. Since $\varepsilon \in W^{m+1, \frac{2 m}{m-1}, 1} \cap L^{\infty}\left(B_{1 / 2}^{2 m}\right), P \in W^{m, 2} \cap L^{\infty}\left(B_{1 / 2}^{2 m}\right)$ and $\Delta^{m-1} u_{r} \in W^{2-m, 2}\left(B_{1 / 2}^{2 m}\right)$ we have

$$
(i d+\varepsilon) P \Delta^{m-1} u \in W^{2-m, 2}\left(B_{1 / 2}^{2 m}\right) .
$$

Set $f=(i d+\varepsilon) P \Delta^{m-1} u$. Then

$$
-\Delta f=\delta C \quad \text { on } B_{1 / 2}^{2 m} \text {. }
$$

By Theorem 6.2 in [4] we get $f \in W^{3-m, \frac{2 m}{m+1}, 1}\left(B_{\lambda}\right)$ on a smaller ball with radius $0<\lambda<$ $1 / 2$. Since $(i d+\varepsilon) P$ is invertible we rewrite (4.19)

$$
\Delta^{m-1} u=[(i d+\varepsilon) P]^{-1} f
$$

and $\Delta^{m-1} u \in W^{3-m, \frac{2 m}{m+1}, 1}\left(B_{\lambda}^{2 m}\right)$. But this means $u \in W^{m+1, \frac{2 m}{m+1}, 1}\left(B_{\lambda}^{2 m}\right)$ and $W^{m+1, \frac{2 m}{m+1}, 1}$ $\left(B_{\lambda}^{2 m}\right) \hookrightarrow C^{0}\left(B_{\lambda}^{2 m}\right)$ (see Theorem 2.3 in [5]).

Up until now we have assumed that $\sigma$ is arbitrarily small so that it satisfies the assumptions of Theorem 4.1 and the fixed point argument. A priori this is not true for components $V_{k}, w_{k}$ of a system of the form (2.4). However any solution $u$ is continuous. To see this we rescale $u$ (see [4] for a detailed proof). Let $x_{0} \in B^{2 m}$ and $r>0$ small enough so that $u_{r}: B^{2 m} \rightarrow \mathbb{R}^{n}, u_{r}(x):=u\left(x_{0}+r x\right)$ is a solution of (2.4) on $B^{2 m}$ with corresponding rescaled components $V_{k, r}$ and $w_{k, r}$,

$$
\begin{aligned}
\sigma_{r}:= & \sum_{k=0}^{m-2}\left\|w_{k, r}\right\|_{W^{2 k+2-m, 2}\left(B^{2 m}\right)}+\sum_{k=1}^{m-1}\left\|V_{k, r}\right\|_{W^{2 k+1-m, 2}\left(B^{2 m}\right)} \\
& +\left\|\eta_{r}\right\|_{W^{2-m, 2}\left(B^{2 m}\right)}+\left\|F_{r}\right\|_{W^{2-m, \frac{2 m}{m+1}, 1}\left(B^{2 m}\right)},
\end{aligned}
$$


$\sigma_{r}<\sigma_{0}$ and $B_{r}^{2 m}\left(x_{0}\right) \subset B^{2 m}$. By the above we have $u_{r} \in C^{0}\left(B_{\lambda}^{2 m}\right)$ which is the same as $u \in C^{0}\left(B_{r \lambda}^{2 m}\left(x_{0}\right)\right)$. A simple covering argument yields $u \in C^{0}\left(B^{2 m}\right)$.

Funding Open Access funding enabled and organized by Projekt DEAL.

Open Access This article is licensed under a Creative Commons Attribution 4.0 International License, which permits use, sharing, adaptation, distribution and reproduction in any medium or format, as long as you give appropriate credit to the original author(s) and the source, provide a link to the Creative Commons licence, and indicate if changes were made. The images or other third party material in this article are included in the article's Creative Commons licence, unless indicated otherwise in a credit line to the material. If material is not included in the article's Creative Commons licence and your intended use is not permitted by statutory regulation or exceeds the permitted use, you will need to obtain permission directly from the copyright holder. To view a copy of this licence, visit http://creativecommons.org/licenses/by/4.0/.

\section{References}

1. Angelsberg, Gilles, Pumberger, D.: A regularity result for polyharmonic maps with higher integrability. Ann. Global Anal. Geom. 35(1), 63-81 (2009)

2. Bethuel, F., Ghidaglia, J.-M.: Improved regularity of solutions to elliptic equations involving Jacobians and applications. J. Math. Pures Appl. 72(5), 441-474 (1993)

3. Chang, Sun-Yung A., Wang, Lihe, Yang, Paul C.: A regularity theory of biharmonic maps. Comm. Pure Appl. Math. 52(9), 1113-1137 (1999)

4. de Longueville, Frédéric Louis.: Regularität der Lösungen von Systemen (2m)-ter Ordnung vom polyharmonischen Typ in kritischer Dimension, https://doi.org/10.17185/duepublico/70153

5. de Longueville, Frédéric Louis., Gastel, Andreas.: Conservation laws for even order systems of polyharmonic map type, arXiv:1909:05697

6. Gastel, Andreas, Scheven, C.: Regularity of polyharmonic maps in the critical dimension. Comm. Anal. Geom. 17(2), 185-226 (2009)

7. Giaquinta, Mariano., Martinazzi, Luca.: An introduction to the regularity theory for elliptic systems, harmonic maps and minimal graphs, volume 11 of Appunti. Scuola Normale Superiore di Pisa (Nuova Serie) [Lecture Notes. Scuola Normale Superiore di Pisa (New Series)]. Edizioni della Normale, Pisa, second edition, (2012)

8. Grafakos, Loukas: Classical Fourier analysis. In: Axler, Sheldon, Ribet, Kenneth (eds.) Graduate Texts in Mathematics, vol. 249, 2nd edn. Springer, New York (2008)

9. Guo, Chang-Yu., Xiang, Chang-Lin.: Regularity of weak solutions to higher order elliptic systems in critical dimensions, arXiv:2010.09149

10. Hélein, Frédéric.: Régularité des applications faiblement harmoniques entre une surface et une variété riemannienne. C. R. Acad. Sci. Paris Sér. I Math. 312(8), 591-596 (1991)

11. Hélein, Frédéric: Harmonic maps, conservation laws and moving frames. In: Bollobas, B., Fulton, W., Katok, A., Kirwan, F., Sarnak, P. (eds.) Cambridge Tracts in Mathematics, vol. 150. Cambridge University Press, Cambridge (2002)

12. Hunt, Richard A.: On $L(p, q)$ spaces. Enseign. Math. 2(12), 249-276 (1966)

13. Jost, Jürgen: Riemannian geometry and geometric analysis, 7th edn. Springer, Cham (2017)

14. Lamm, Tobias, Riviere, T.: Conservation laws for fourth order systems in four dimensions. Comm. Partial Differ. Equ. 33(1-3), 245-262 (2008)

15. Rivière, Tristan: Conservation laws for conformally invariant variational problems. Invent. Math. 168(1), $1-22(2007)$

16. Rivière, Tristan.: Conformally invariant variational problems, arXiv:1206.2116

17. Schikorra, Armin: A remark on gauge transformations and the moving frame method. Ann. Inst. H. Poincaré Anal. Non Linéaire 27(2), 503-515 (2010)

18. Struwe, Michael: Partial regularity for biharmonic maps, revisited. Calc. Var. Partial Differ. Equ. 33(2), 249-262 (2008)

19. Tartar, Luc: Imbedding theorems of Sobolev spaces into Lorentz spaces. Boll. Unione Mat. Ital. Sez. B Artic. Ric. Mat. (8) 1(3), 479-500 (1998)

20. Uhlenbeck, Karen K.: Connections with $L^{p}$ bounds on curvature. Comm. Math. Phys. 83(1), 31-42 (1982)

21. Wang, Changyou: Biharmonic maps from $R^{4}$ into a Riemannian manifold. Math. Z. 247(1), 65-87 (2004) 
22. Wente, H.C.: An existence theorem for surfaces of constant mean curvature. J. Math. Anal. Appl. 26, 318-344 (1969)

23. Ziemer, WP.: Weakly differentiable functions. Springer, New York (1989)

Publisher's Note Springer Nature remains neutral with regard to jurisdictional claims in published maps and institutional affiliations. 